\title{
Germline-epigenome crosstalk promotes aggressive subsets of prostate tumours
}

$14 \%$ of known
prostate
cancer risk
loci might
influence
cancer risk
by modulating
tumour
methylation

Germline and epigenetic crosstalk in primary prostate tumours could help to identify biomarkers of aggressive disease to improve patient stratification and optimize therapy assignation, according to new data published in Nature Medicine.

A number of factors contribute to prostate oncogenesis, including germline, epigenetic and environmental changes, which ultimately result in the dysregulation of cellular processes that is characteristic of malignancy. Genome-wide association studies (GWAS) have identified $>100$ germline variants associated with an increased risk of cancer diagnosis. However, although these data suggest that germline single-nucleotide polymorphisms (SNPs) could cause development of aggressive tumours, GWAS have failed to identify robust associations with specific loci; modulation of the epigenome via SNPs might explain this discrepancy and provide the missing link. Some germline alterations in tumour-suppressor genes - such as RB1, APC, BRCA1 and $B R C A 2$ - can induce epigenomic changes, which can further affect oncogenesis. Tumours are able to hijack epigenetic regulatory systems to silence tumour suppressors, rewiring DNA methylation. Enrichment of susceptibility loci at regulatory regions can modulate the epigenome, increasing tumour aggressiveness. Thus, co-analysis of the germ line and methylation might identify loci involved in tumorigenesis that did not previously reach significance that could provide biomarkers and inform the development of aggressive disease.

Houlahan and colleagues began by analysing 241 patients with treatment-naive prostate cancer (discovery cohort) who underwent germline whole-genome sequencing (WGS) and methylation profiling. Their aim was to quantify polymorphisms that modulate specific epigenetic features, termed cis-methylation quantitative trait loci (meQTLs). They then further investigated the tumour meQTLs - loci associated with altered methylation in malignant tissue but not in benign tissue - to distinguish them from meQTLs that exert effects in normal epithelial tissue. Validation using data from 348 patients in The Cancer Genome Atlas (TCGA) demonstrated that $14 \%$ of known prostate cancer risk loci might influence cancer risk by modulating tumour methylation. Some tumour meQTLs targeted prognostic cancer driver genes, including $A K T 1$, the meQTL for which was predictive of aggressive disease in both the discovery cohort (HR 2.85; $P=0.0058)$ and validation cohort (HR 2.2; $P=0.017$ ).

Overall, the team identified and validated 1,178 tumour meQTLs, 17 of which showed tumour-specific RNA or protein abundance changes (which were termed tumour meQTLexpression QTLs; eQTLs). These tumour meQTLs were enriched at tumour-specific regulatory regions in prostate cancer cell lines (LNCaP, $\mathrm{PC} 3, \mathrm{VCaP}$ and 22Rv1), a prostate epithelial cell line (RWPE-1) and in primary tumours, and preferentially targeted sites of chromatin looping.

The team identified a number of loci that were predictive of aggressive disease. Of note, their investigations identified the rs684232 haplotype, which is a known risk locus, providing validation for their approach, and loci targeting prostate cancer driver events, such as TCERG1L and AKT1.
However, not all the identified risk meQTLs were tumour specific, and the authors hypothesize that the meQTLs detected in reference tissue might facilitate tumour initiation by modulating preneoplastic methylation, whereas the tumour meQTLs might facilitate tumour progression by modulating oncogenic methylation.

"The tumour-specific role of some germline variants in modulating tumour methylation is particularly interesting, as these germline variants are present in nonmalignant tissue but their regulatory role appears to only be triggered in a tumourigenic environment," first author Katie Houlahan tells Nature Reviews Urology. "Thus the tumourspecific label might stratify germline variants that are involved in tumour initiation from those involved in tumour progression. This is just a hypothesis at this point and requires more follow-up."

Corresponding author Paul Boutros summarizes: "The germline genome a patient is born with influences the ways that prostate cancer evolves in literally thousands of ways. These influences can start off at the epigenome and then ripple through the central dogma to affect cellular biology. Those changes in cellular biology seem to, in some not yet understood ways, influence clinical behaviour — including a patient's risk of dying from the disease. Taken together, this means our work reveals a new landscape of mechanisms by which germline features influence prostate cancer behaviour."

Annette Fenner

ORIGINAL ARTICLE Houlahan, K. E. et al. Genome-wide germline correlates of the epigenetic landscape of prostate cancer. Nat. Med. 25, 1615-1626 (2019) 